\title{
On the algebra $\mathcal{A}_{\hbar, \eta}\left(\operatorname{osp}(2 \mid 2)^{(2)}\right)$ and free boson representations
}

\author{
Niall MacKay ${ }^{1 *}$ and Liu Zhao ${ }^{1,2 \dagger \dagger}$ \\ ${ }^{1}$ Department of Mathematics, University of York, York YO10 5DD, UK \\ ${ }^{2}$ Institute of Modern Physics, Northwest University, Xian 710069, China
}

\begin{abstract}
A two-parameter quantum deformation of the affine Lie super algebra $\operatorname{osp}(2 \mid 2)^{(2)}$ is introduced and studied in some detail. This algebra is the first example associated with nonsimply-laced and twisted root systems of a quantum current algebra with the structure of a so-called infinite Hopf family of (super)algebras. A representation of this algebra at $c=1$ is realized in the product Fock space of two commuting sets of Heisenberg algebras.
\end{abstract}

\section{Introduction}

The study of two-parameter deformations of affine Lie (super)algebras has turned out to be quite fruitful in the last few years. These algebras are in a sense deformations of the standard quantum affine algebras or Yangian doubles which all have the structure of quasi-triangular Hopf algebras. In contrast, the two-parameter deformations are not Hopf algebras, but rather have twisted Hopf structures, one of which is the Drinfeld twist of Hopf algebras or quasi-Hopf algebras, while the other is the infinite Hopf family of (super)algebras. So far, the relationship between the two generalized co-structures remains ill-understood.

From the physical point of view, the above two classes of two-parameter deformations appear in different contexts. Quasi-Hopf algebras [1] occur in the study of symmetries of face- and vertextype models of statistical mechanics [6], and are closely related to the face-type Boltzmann weights (Yang-Baxter R-matrix), while infinite Hopf families of (super)algebras [4, 5] occur only in the representation theory of quantum deformed Virasoro and W-algebras [2] (and act as the algebra of screening currents), which in turn are algebras characterizing the dynamical symmetries of certain massive integrable quantum field theories [7].

\footnotetext{
*e-mail: nm15@york.ac.uk

${ }^{\dagger}$ e-mail: lzhao@phy.nwu.edu.cn

${ }^{\ddagger}$ Royal Society visiting fellow
} 
Despite the great deal of work that has been done on these two-parameter deformations, many problems remain unsolved. In particular, for the second class of two-parameter deformations (the infinite Hopf families) nothing has been said concerning root systems of nonsimply-laced and/or twisted types. For super root systems, the only case which has been considered is the case of $\operatorname{osp}(1 \mid 2)^{(1)}$ [9]. In this article, therefore, we aim to provide more concrete examples of this kind, and in particular the trigonometric two-parameter deformation of $\operatorname{ssp}(2 \mid 2)^{(2)}$. This algebra is based on a root system which is simultaneously non-simply laced, twisted and super.

\section{Definition and structure of the algebra $\mathcal{A}_{\hbar, \eta}\left(\operatorname{osp}(2 \mid 2)^{(2)}\right)$}

\subsection{Definition of $\mathcal{A}_{\hbar, \eta}\left(\operatorname{osp}(2 \mid 2)^{(2)}\right)$.}

We start with the definition of the algebra $\mathcal{A}_{\hbar, \eta}\left(\operatorname{osp}(2 \mid 2)^{(2)}\right)$. The notation follows that of $\mathcal{A}_{\hbar, \eta}(\hat{g})$ [0, 田, 司] and $\mathcal{A}_{\hbar, \eta}\left(\operatorname{osp}(1 \mid 2)^{(1)}\right)[9]$.

Definition 2.1 The algebra $\mathcal{A}_{\hbar, \eta}\left(\operatorname{osp}(2 \mid 2)^{(2)}\right)$, considered as a continuously distributed current superalgebra, is a $Z_{2}$ graded associative algebra over $C$ generated by the currents $E(u), F(u)$, $H^{ \pm}(u)$, the central element $c$ and unit 1 with parities $\pi[E(u)]=\pi[F(u)]=1, \pi\left[H^{ \pm}(u)\right]=\pi[c]=$ $\pi[1]=0$ and generating relations

$$
\begin{aligned}
E(u) E(v) & =-\frac{\cosh [\pi \eta(u-v+i \hbar)]}{\cosh [\pi \eta(u-v-i \hbar)]} E(v) E(u) \\
F(u) F(v) & =-\frac{\cosh \left[\pi \eta^{\prime}(u-v-i \hbar)\right]}{\cosh \left[\pi \eta^{\prime}(u-v+i \hbar)\right]} F(v) F(u) \\
H^{ \pm}(u) E(v) & =\frac{\cosh [\pi \eta(u-v+i \hbar \pm i \hbar c / 4)]}{\cosh [\pi \eta(u-v-i \hbar \pm i \hbar c / 4)]} E(v) H^{ \pm}(u) \\
H^{ \pm}(u) F(v) & =\frac{\cosh \left[\pi \eta^{\prime}(u-v-i \hbar \mp i \hbar c / 4)\right]}{\cosh \left[\pi \eta^{\prime}(u-v+i \hbar \mp i \hbar c / 4)\right]} F(v) H^{ \pm}(u) \\
H^{ \pm}(u) H^{ \pm}(v) & =\frac{\cosh [\pi \eta(u-v+i \hbar)]}{\cosh [\pi \eta(u-v-i \hbar)]} \frac{\cosh \left[\pi \eta^{\prime}(u-v-i \hbar)\right]}{\cosh \left[\pi \eta^{\prime}(u-v+i \hbar)\right]} H^{ \pm}(v) H^{ \pm}(u), \\
H^{+}(u) H^{-}(v) & =\frac{\cosh [\pi \eta(u-v+i \hbar+i \hbar c / 2)]}{\cosh [\pi \eta(u-v-i \hbar+i \hbar c / 2)]} \frac{\cosh \left[\pi \eta^{\prime}(u-v-i \hbar-i \hbar c / 2)\right]}{\cosh \left[\pi \eta^{\prime}(u-v+i \hbar-i \hbar c / 2)\right]} H^{-}(v) H^{+}(u), \\
\{E(u), F(v)\} & =\frac{2 \pi}{\hbar}\left[\delta(u-v-i \hbar c / 2) H^{+}(u-i \hbar c / 4)-\delta(u-v+i \hbar c / 2) H^{-}(v-i \hbar c / 4)\right]
\end{aligned}
$$

where

$$
\frac{1}{\eta^{\prime}}-\frac{1}{\eta}=\hbar c
$$

and $\hbar$ and $\eta$ are generic deformation parameters.

For later reference, we denote the subalgebras generated respectively by the currents $\{E(u)\}$ and $\{F(u)\}$ as $\mathcal{N}_{ \pm}\left[\mathcal{A}_{\hbar, \eta}\left(\operatorname{osp}(2 \mid 2)^{(2)}\right)\right]$. 
It is interesting to compare the generating relations of the algebra $\mathcal{A}_{\hbar, \eta}\left(\operatorname{osp}(2 \mid 2)^{(2)}\right)$ and those of the quantum affine super algebra $U_{q}\left(\operatorname{osp}(2 \mid 2)^{(2)}\right)$. The latter has the generating relations [B]

$$
\begin{aligned}
X^{+}(z) X^{+}(w) & =-\frac{z q+w}{z+w q} X^{+}(w) X^{+}(z), \\
X^{-}(z) X^{-}(w) & =-\frac{z+w q}{z q+w} X^{-}(w) X^{-}(w), \\
\psi^{ \pm}(z) X^{+}(w) & =\frac{z_{ \pm} q+w}{z_{ \pm}+w q} X^{+}(w) \psi^{ \pm}(z), \\
\psi^{ \pm}(z) X^{-}(w) & =\frac{z_{\mp}+w q}{z_{\mp} q+w} X^{-}(w) \psi^{ \pm}(z), \\
\psi^{ \pm}(z) \psi^{ \pm}(w) & =\psi^{ \pm}(w) \psi^{ \pm}(z), \\
\psi^{+}(z) \psi^{-}(w) & =\frac{z_{+} q+w_{-}}{z_{+}+w_{-} q} \frac{1}{z_{-} q+w_{+} q} \psi_{+}^{-}(w) \psi^{+}(z), \\
\left\{X^{+}(z), X^{-}(w)\right\} & =\frac{1}{\left(q-q^{-1}\right) z w}\left[\delta\left(\frac{w}{z} \gamma\right) \psi^{+}\left(z_{-}\right)-\delta\left(\frac{w}{z} \gamma^{-1}\right) \psi^{-}\left(w_{-}\right)\right],
\end{aligned}
$$

where' $z_{ \pm}=z \gamma^{ \pm 1 / 2}$. Notice that the $\delta$-functions appearing in (7) and (14) are supported differently, the former at 0 (i.e. the standard Dirac $\delta$-function), the latter at $1\left(\delta(z) \equiv \sum_{n \in Z} z^{n}\right.$ as a formal power series). We also denote the subalgebras of $U_{q}\left(\operatorname{osp}(2 \mid 2)^{(2)}\right)$ generated respectively by $X^{+}(z)$ and $X^{-}(z)$ by $\mathcal{N}_{ \pm}\left[U_{q}\left(\operatorname{osp}(2 \mid 2)^{(2)}\right)\right]$.

The following two propositions justify the similarities between the two algebras $\mathcal{A}_{\hbar, \eta}\left(\operatorname{osp}(2 \mid 2)^{(2)}\right)$ and $U_{q}\left(\operatorname{osp}(2 \mid 2)^{(2)}\right)$ : first,

Proposition 2.2 There are algebra homomorphisms $\rho^{+}: \mathcal{N}_{+}\left[\mathcal{A}_{\hbar, \eta}\left(\operatorname{osp}(2 \mid 2)^{(2)}\right)\right] \rightarrow \mathcal{N}_{+}\left[U_{q}\left(\operatorname{osp}(2 \mid 2)^{(2)}\right)\right]$, $\rho^{-}: \mathcal{N}_{-}\left[\mathcal{A}_{\hbar, \eta}\left(\operatorname{osp}(2 \mid 2)^{(2)}\right)\right] \rightarrow \mathcal{N}_{-}\left[U_{q^{\prime}}\left(\operatorname{osp}(2 \mid 2)^{(2)}\right)\right]$, where under $\rho^{ \pm}$, the parameters behave as

$$
\begin{aligned}
& \rho^{+}\left(e^{2 \pi \eta u}\right)=z, \\
& \rho^{+}\left(e^{2 \pi i \eta \hbar}\right)=q,
\end{aligned}
$$

and

$$
\begin{aligned}
& \rho^{-}\left(e^{2 \pi \eta^{\prime} u}\right)=z, \\
& \rho^{-}\left(e^{2 \pi i \eta^{\prime} \hbar}\right)=q^{\prime},
\end{aligned}
$$

respectively.

Recalling that $\eta$ and $\eta^{\prime}$ are different only when $c \neq 0$, we also have

\footnotetext{
${ }^{1}$ In the original presentation of $U_{q}\left(\operatorname{osp}(2 \mid 2)^{(2)}\right)$ in $\left.\mathbb{8}\right]$, the element $\gamma$ was written as $q^{c}$. However, to avoid confusion with the central element $c$ of the algebra $\mathcal{A}_{\hbar, \eta}\left(\operatorname{osp}(2 \mid 2)^{(2)}\right)$, we intentionally rename it $\gamma$, as is usual in ordinary quantum affine algebras.
} 
Proposition 2.3 There is an algebra homomorphism between $\mathcal{A}_{\hbar, \eta}\left(\operatorname{osp}(2 \mid 2)^{(2)}\right)$ at $c=0$ and $U_{q}\left(\operatorname{osp}(2 \mid 2)^{(2)}\right)$ at $\gamma=1$ :

$$
\begin{aligned}
\mathcal{E}: \mathcal{A}_{\hbar, \eta}\left(\operatorname{osp}(2 \mid 2)^{(2)}\right) & \rightarrow U_{q}\left(\operatorname{osp}(2 \mid 2)^{(2)}\right), \\
E(u) & \longmapsto \sqrt{2} z X^{+}(z), \\
F(u) & \longmapsto \sqrt{2} z X^{-}(z), \\
\frac{2 \pi}{\hbar} H^{ \pm}(u) & \longmapsto \frac{1}{q-q^{-1}} \psi^{ \pm}(z),
\end{aligned}
$$

where $z=e^{2 \pi \eta \hbar u}, q=e^{2 \pi i \eta \hbar}$.

Proposition 2.2 indicates that the algebra $\mathcal{A}_{\hbar, \eta}\left(\operatorname{osp}(2 \mid 2)^{(2)}\right)$ is actually an interpolation between (Borel subalgebras of) two standard quantum affine algebras $U_{q}\left(\operatorname{osp}(2 \mid 2)^{(2)}\right)$ and $U_{q^{\prime}}\left(\operatorname{osp}(2 \mid 2)^{(2)}\right)$ with different deformation parameters, while Proposition 2.3 further states that at $c=0$, the algebra $\mathcal{A}_{\hbar, \eta}\left(\operatorname{osp}(2 \mid 2)^{(2)}\right)$ degenerates into $U_{q}\left(\operatorname{osp}(2 \mid 2)^{(2)}\right)$ at $\gamma=1$.

\subsection{Co-structure}

As expected, this algebra possesses the structure of an infinite Hopf family of super algebras, whose definition can be found in [9] (see also [由, 5]). In fact, if we denote $\mathcal{A}_{n}=\mathcal{A}_{\hbar, \eta^{(n)}}\left(\operatorname{osp}(2 \mid 2)^{(2)}\right)_{c_{n}}$ where $\eta^{(n)}$ is defined iteratively via $\frac{1}{\eta^{(n+1)}}-\frac{1}{\eta^{(n)}}=\hbar c_{n}$ starting from $\eta^{(1)}=\eta$ and taking $c_{n} \in Z \backslash Z_{-}$, we can define the following co-structures over the family of algebras $\left\{\mathcal{A}_{n}, n \in Z\right\}$ :

- the comultiplications $\Delta_{n}^{ \pm}$(algebra homomorphisms $\Delta_{n}^{+}: \mathcal{A}_{n} \rightarrow \mathcal{A}_{n} \otimes \mathcal{A}_{n+1}, \Delta_{n}^{-}: \mathcal{A}_{n} \rightarrow$ $\left.\mathcal{A}_{n-1} \otimes \mathcal{A}_{n}\right)$ :

$$
\begin{aligned}
\Delta_{n}^{+} c_{n} & =c_{n}+c_{n+1} \\
\Delta_{n}^{-} c_{n} & =c_{n-1}+c_{n}, \\
\Delta_{n}^{+} H^{+}\left(u ; \eta^{(n)}\right) & =H^{+}\left(u+\frac{i \hbar c_{n+1}}{4} ; \eta^{(n)}\right) \otimes H^{+}\left(u-\frac{i \hbar c_{n}}{4} ; \eta^{(n+1)}\right), \\
\Delta_{n}^{-} H^{+}\left(u ; \eta^{(n)}\right) & =H^{+}\left(u+\frac{i \hbar c_{n}}{4} ; \eta^{(n-1)}\right) \otimes H^{+}\left(u-\frac{i \hbar c_{n-1}}{4} ; \eta^{(n)}\right), \\
\Delta_{n}^{+} H^{-}\left(u ; \eta^{(n)}\right) & =H^{-}\left(u-\frac{i \hbar c_{n+1}}{4} ; \eta^{(n)}\right) \otimes H^{-}\left(u+\frac{i \hbar c_{n}}{4} ; \eta^{(n+1)}\right), \\
\Delta_{n}^{-} H^{-}\left(u ; \eta^{(n)}\right) & =H^{-}\left(u-\frac{i \hbar c_{n}}{4} ; \eta^{(n-1)}\right) \otimes H^{-}\left(u+\frac{i \hbar c_{n-1}}{4} ; \eta^{(n)}\right), \\
\Delta_{n}^{+} E\left(u ; \eta^{(n)}\right) & =E\left(u ; \eta^{(n)}\right) \otimes 1+H^{-}\left(u+\frac{i \hbar c_{n}}{4} ; \eta^{(n)}\right) \otimes E\left(u+\frac{i \hbar c_{n}}{2} ; \eta^{(n+1)}\right), \\
\Delta_{n}^{-} E\left(u ; \eta^{(n)}\right) & =E\left(u ; \eta^{(n-1)}\right) \otimes 1+H^{-}\left(u+\frac{i \hbar c_{n-1}}{4} ; \eta^{(n-1)}\right) \otimes E\left(u+\frac{i \hbar c_{n-1}}{2} ; \eta^{(n)}\right), \\
\Delta_{n}^{+} F\left(u ; \eta^{(n)}\right) & =1 \otimes F\left(u ; \eta^{(n+1)}\right)+F\left(u+\frac{i \hbar c_{n+1}}{2} ; \eta^{(n)}\right) \otimes H^{+}\left(u+\frac{i \hbar c_{n+1}}{4} ; \eta^{(n+1)}\right), \\
\Delta_{n}^{-} F\left(u ; \eta^{(n)}\right) & =1 \otimes F\left(u ; \eta^{(n)}\right)+F\left(u+\frac{i \hbar c_{n}}{2} ; \eta^{(n-1)}\right) \otimes H^{+}\left(u+\frac{i \hbar c_{n}}{4} ; \eta^{(n)}\right) ;
\end{aligned}
$$


- the counits $\epsilon_{n}$ (algebra homomorphism $\epsilon_{n}: \mathcal{A}_{n} \rightarrow C$ ) :

$$
\begin{aligned}
\epsilon_{n}\left(c_{n}\right) & =0, \\
\epsilon_{n}\left(1_{n}\right) & =1, \\
\epsilon_{n}\left(H_{i}^{ \pm}\left(u ; \eta^{(n)}\right)\right) & =1, \\
\epsilon_{n}\left(E_{i}\left(u ; \eta^{(n)}\right)\right) & =0, \\
\epsilon_{n}\left(F_{i}\left(u ; \eta^{(n)}\right)\right) & =0 ;
\end{aligned}
$$

- the antipodes $S_{n}^{ \pm}$(algebra anti-homomorphisms $S_{n}^{ \pm}: \mathcal{A}_{n} \rightarrow \mathcal{A}_{n \pm 1}$ ):

$$
\begin{aligned}
S_{n}^{ \pm}\left(c_{n}\right) & =-c_{n \pm 1}, \\
S_{n}^{ \pm}\left(H^{ \pm}\left(u ; \eta^{(n)}\right)\right) & =\left[H^{ \pm}\left(u ; \eta^{(n \pm 1)}\right)\right]^{-1}, \\
S_{n}^{ \pm}\left(E\left(u ; \eta^{(n)}\right)\right) & =-H^{-}\left(u-\frac{i \hbar c_{n \pm 1}}{4} ; \eta^{(n \pm 1)}\right)^{-1} E\left(u-\frac{i \hbar c_{n \pm 1}}{2} ; \eta^{(n \pm 1)}\right), \\
S_{n}^{ \pm}\left(F\left(u ; \eta^{(n)}\right)\right) & =-F\left(u-\frac{i \hbar c_{n \pm 1}}{2} ; \eta^{(n \pm 1)}\right) H^{+}\left(u-\frac{i \hbar c_{n \pm 1}}{4} ; \eta^{(n \pm 1)}\right)^{-1},
\end{aligned}
$$

where $\otimes$ stands for the direct super product defined by

$$
(A \otimes B)(C \otimes D)=(-1)^{\pi[B] \pi[C]} A B \otimes C D
$$

for homogeneous elements $A, B, C, D$. It is a trivial (but tedious) exercise to check that these structures satisfy all the defining axioms of an infinite Hopf family of super algebras,

- $\left(\epsilon_{n} \otimes i d_{n+1}\right) \circ \Delta_{n}^{+}=\tau_{n}^{+},\left(i d_{n-1} \otimes \epsilon_{n}\right) \circ \Delta_{n}^{-}=\tau_{n}^{-}$

- $m_{n+1} \circ\left(S_{n}^{+} \otimes i d_{n+1}\right) \circ \Delta_{n}^{+}=\epsilon_{n+1} \circ \tau_{n}^{+}, m_{n-1} \circ\left(i d_{n-1} \otimes S_{n}^{-}\right) \circ \Delta_{n}^{-}=\epsilon_{n-1} \circ \tau_{n}^{-}$

- $\left(\Delta_{n}^{-} \otimes i d_{n+1}\right) \circ \Delta_{n}^{+}=\left(i d_{n-1} \otimes \Delta_{n}^{+}\right) \circ \Delta_{n}^{-}$

where $m_{n}$ is the (super)multiplication for $\mathcal{A}_{n}$ and $\tau_{n}^{ \pm}$are algebra shift morphisms $\tau_{n}^{ \pm}: \mathcal{A}_{n} \rightarrow$ $\mathcal{A}_{n \pm 1}$ which obeys $\tau_{n+1}^{-} \tau_{n}^{+}=i d_{n}=\tau_{n-1}^{+} \tau_{n}^{-}$.

The operations $\Delta_{n}^{ \pm}$are related to each other by the shift morphisms:

$$
\begin{aligned}
& \Delta_{n}^{-}=\left(\tau_{n}^{-} \otimes \tau_{n+1}^{-}\right) \circ \Delta_{n}^{+}, \\
& \Delta_{n}^{+}=\left(\tau_{n-1}^{+} \otimes \tau_{n}^{+}\right) \circ \Delta_{n}^{-} .
\end{aligned}
$$

Thus the easily-observed co-commutativity between the two co-multiplications

$$
\left(\Delta_{n}^{-} \otimes i d_{n+1}\right) \circ \Delta_{n}^{+}=\left(i d_{n-1} \otimes \Delta_{n}^{+}\right) \circ \Delta_{n}^{-}
$$


can be rewritten in terms of only one of the two co-multiplications, and turns out to become a statement of the non-coassociativity of the co-multiplications:

$$
\begin{aligned}
& {\left[\left(\left(\tau_{n}^{-} \otimes \tau_{n+1}^{-}\right) \circ \Delta_{n}^{+}\right) \otimes i d_{n+1}\right] \circ \Delta_{n}^{+}=\left(i d_{n-1} \otimes \Delta_{n}^{+}\right) \circ\left(\left(\tau_{n}^{-} \otimes \tau_{n+1}^{-}\right) \circ \Delta_{n}^{+}\right),} \\
& \left(\Delta_{n}^{-} \otimes i d_{n+1}\right) \circ\left(\left(\tau_{n-1}^{+} \otimes \tau_{n}^{+}\right) \circ \Delta_{n}^{-}\right)=\left[i d_{n-1} \otimes\left(\left(\tau_{n-1}^{+} \otimes \tau_{n}^{+}\right) \circ \Delta_{n}^{-}\right)\right] \circ \Delta_{n}^{-} .
\end{aligned}
$$

Notice that these twisted co-associativity conditions are different from that of the Drinfeld twists. However, the effects of these two different kinds of twists are the same: they all allow one to construct fused (tensor product) representations for the algebras under investigation, although the co-structures are not co-associative.

Now recall that definition 2.1 defines the algebra $\mathcal{A}_{\hbar, \eta}\left(\operatorname{osp}(2 \mid 2)^{(2)}\right)$ only as a formal algebra, in the sense that all currents thus defined are actually only distributions. To assign precise meaning to the algebra $\mathcal{A}_{\hbar, \eta}\left(\operatorname{osp}(2 \mid 2)^{(2)}\right)$ we need to specify the actual generators and relations, and this can be done only separately for two distinct cases $c=0$ and $c \neq 0$ (as in the case of $\mathcal{A}_{\hbar, \eta}\left(\widehat{s l_{2}}\right)$ [7] and $\mathcal{A}_{\hbar, \eta}(\hat{g})$ 田). For details, the reader is directed to Khoroshkin et al. [7] in the $\widehat{s l}_{2}$ case. The present case is in complete analogy.

\section{$3 \quad$ Representation theory}

\subsection{Case $c=0$}

Recall that for $c=0$, there is an algebra homomorphism between the algebras $\mathcal{A}_{\hbar, \eta}\left(\operatorname{osp}(2 \mid 2)^{(2)}\right)$ and $U_{q}\left(\operatorname{osp}(2 \mid 2)^{(2)}\right)$ for $q=e^{2 \pi i \eta \hbar}$. Thus the evaluation representation of $U_{q}\left(\operatorname{osp}(2 \mid 2)^{(2)}\right)$ presented in [8] can be extended into an evaluation representation of $\mathcal{A}_{\hbar, \eta}\left(\operatorname{osp}(2 \mid 2)^{(2)}\right)$ in terms of the evaluation homomorphism $\mathcal{E}$. This evaluation representation justifies the relationship between the algebra $\mathcal{A}_{\hbar, \eta}\left(\operatorname{osp}(2 \mid 2)^{(2)}\right)$ and the root system of type $\operatorname{osp}(2 \mid 2)^{(2)}$.

\subsection{Case $c=1$ and structure of the Fock space}

As usual, the tool we need to construct a representation of $\mathcal{A}_{\hbar, \eta}\left(\operatorname{osp}(2 \mid 2)^{(2)}\right)$ at $c=1$ is the free boson realization. Throughout this subsection we have $1 / \eta^{\prime}=1 / \eta+\hbar$.

Define the Heisenberg algebras $\mathcal{H}_{\alpha}, \mathcal{H}_{\beta}$ respectively by

$$
\begin{aligned}
{[\alpha(\lambda), \alpha(\mu)] } & =A(\lambda) \delta(\lambda+\mu), \\
{[\beta(\lambda), \beta(\mu)] } & =B(\lambda) \delta(\lambda+\mu), \\
{[\alpha(\lambda), \beta(\mu)] } & =0, \\
(\lambda & \neq \mu)
\end{aligned}
$$

where $A(\lambda)$ and $B(\lambda)$ are given as

$$
A(\lambda)=\frac{\lambda}{4 \cosh \frac{\hbar \lambda}{2}+\left(\operatorname{csch} \frac{\lambda}{2 \eta}-\operatorname{csch} \frac{\lambda}{2 \eta^{\prime}}\right) \sinh \hbar \lambda+2},
$$




$$
B(\lambda)=\frac{\lambda\left(\left(1+\operatorname{csch} \frac{\lambda}{2 \eta} \sinh \hbar \lambda\right)\left(1-\operatorname{csch} \frac{\lambda}{2 \eta^{\prime}} \sinh \hbar \lambda\right)-4 \cosh ^{2} \frac{\hbar \lambda}{2}\right)}{4 \cosh \frac{\hbar \lambda}{2}+\left(\operatorname{csch} \frac{\lambda}{2 \eta}-\operatorname{csch} \frac{\lambda}{2 \eta^{\prime}}\right) \sinh \hbar \lambda+2},
$$

both of which are antisymmetric as $\lambda \rightarrow-\lambda$ and regular as $\lambda \rightarrow 0$. In fact we can easily check that

$$
\begin{aligned}
& A(\lambda)=-A(-\lambda), \\
& B(\lambda)=-B(-\lambda),
\end{aligned}
$$

and

$$
\begin{aligned}
A(\lambda) & \sim \frac{\lambda}{2\left(\eta-\eta^{\prime}\right) \hbar+6}+O\left(\lambda^{3}\right), \\
B(\lambda) & \sim \frac{\left[(1+2 \hbar \eta)\left(1-2 \hbar \eta^{\prime}\right)-4\right] \lambda}{2\left(\eta-\eta^{\prime}\right) \hbar+6}+O\left(\lambda^{3}\right),
\end{aligned}
$$

indicating that the Heisenberg algebras $\mathcal{H}_{\alpha}, \mathcal{H}_{\beta}$ are well-defined even at $\lambda=0$. The conjugates of $\alpha(0)$ and $\beta(0)$ have to be introduced separately, however, as follows. Let $Q_{\alpha}=\alpha(0), Q_{\beta}=\beta(0)$ and their conjugate operators $P_{\alpha}, P_{\beta}$ are defined by the following relations:

$$
\begin{aligned}
& {\left[P_{\alpha}, Q_{\alpha}\right]=1} \\
& {\left[P_{\beta}, Q_{\beta}\right]=1} \\
& {\left[P_{\alpha}, Q_{\beta}\right]=\left[P_{\beta}, Q_{\alpha}\right]=0}
\end{aligned}
$$

Now denoting

$$
\begin{aligned}
& X_{a}(\lambda)=\frac{1}{\hbar \lambda}\left(\operatorname{csch} \frac{\lambda}{2 \eta} \sinh \hbar \lambda+2 \cosh \frac{\hbar \lambda}{2}+1\right), \\
& X_{b}(\lambda)=\frac{1}{\hbar \lambda}\left(\operatorname{csch} \frac{\lambda}{2 \eta^{\prime}} \sinh \hbar \lambda-2 \cosh \frac{\hbar \lambda}{2}-1\right), \\
& Y_{a}(\lambda)=Y_{b}(\lambda)=\frac{1}{\hbar \lambda}
\end{aligned}
$$

we can define

$$
\begin{aligned}
a(\lambda) & =X_{a}(\lambda) \alpha(\lambda)+Y_{a}(\lambda) \beta(\lambda), \\
b(\lambda) & =X_{b}(\lambda) \alpha(\lambda)+Y_{b}(\lambda) \beta(\lambda), \\
(\lambda & \neq \mu)
\end{aligned}
$$

so that the corresponding commutation relations read:

$$
[a(\lambda), a(\mu)]=-\frac{1}{\hbar^{2} \lambda}\left(1+\frac{\sinh \hbar \lambda}{\sinh \frac{\lambda}{2 \eta}}\right) \delta(\lambda+\mu),
$$




$$
\begin{aligned}
{[b(\lambda), b(\mu)] } & =-\frac{1}{\hbar^{2} \lambda}\left(1-\frac{\sinh \hbar \lambda}{\sinh \frac{\lambda}{2 \eta^{\prime}}}\right) \delta(\lambda+\mu), \\
{[a(\lambda), b(\mu)] } & =[b(\lambda), a(\mu)]=\frac{2}{\hbar^{2} \lambda} \cosh \frac{\hbar \lambda}{2} \delta(\lambda+\mu), \\
(\lambda & \neq \mu) .
\end{aligned}
$$

These commutation relations are crucial for the construction of the free boson representation for the algebra $\mathcal{A}_{\hbar, \eta}\left(\operatorname{osp}(2 \mid 2)^{(2)}\right)$ and hence we give them a short name for reference: $\mathcal{H}[a, b]$. Recall that we are dealing with generic deformation parameters, we do not consider the specific values of the parameters at which the above bosonic algebra becomes ill-defined (these include the points at which $\frac{1}{2 \eta}$ is a rational multiple of $\hbar$ ).

Before going into the details of the representation theory, we have to specify the structure of the Fock space on which the free bosonic algebra $\mathcal{H}[a, b]$ acts. Actually, there are infinite many ways to realize the bosonic algebra $\mathcal{H}[a, b]$ in terms of two commuting sets of Heisenberg algebras, and what we outlined above is only one of infinitely many choices.

Denoting respectively by $\mathcal{F}_{\alpha}$ and $\mathcal{F}_{\beta}$ the Fock spaces for the Heisenberg algebras $\mathcal{H}_{\alpha}$ and $\mathcal{H}_{\beta}$, we see that the bosonic algebra $\mathcal{H}[a, b]$ can be realized in a proper subspace $\mathcal{F}[a, b]$ of $\mathcal{F}_{\alpha} \otimes \mathcal{F}_{\beta}$ by actions of the form

$$
\begin{aligned}
\mathcal{F}_{\alpha} \otimes \mathcal{F}_{\beta} \supset & \mathcal{F}[a, b] \ni\left|v_{f_{1}, \ldots, f_{n}, g_{1}, \ldots, g_{m}}\right\rangle=\int_{-\infty}^{-\epsilon} d \lambda_{1} f\left(\lambda_{1}\right) a\left(\lambda_{1}\right) \ldots \int_{-\infty}^{-\epsilon} d \lambda_{n} f\left(\lambda_{n}\right) a\left(\lambda_{n}\right) \\
& \times \int_{-\infty}^{-\epsilon} d \mu_{1} g\left(\mu_{1}\right) b\left(\mu_{1}\right) \ldots \int_{-\infty}^{-\epsilon} d \mu_{m} g\left(\mu_{m}\right) b\left(\mu_{m}\right)|0\rangle_{\alpha} \otimes|0\rangle_{\beta}, \\
& \lambda_{1}, \ldots, \lambda_{n} ; \mu_{1}, \ldots, \mu_{m}<0, \forall n, m \in Z, \\
& 0<\epsilon \rightarrow 0^{+} .
\end{aligned}
$$

Notice that the ordering of $a$ and $b$ in the above expression is irrelevant, because all values of $\lambda_{1}, \ldots, \lambda_{n}$ and $\mu_{1}, \ldots, \mu_{m}$ are negative. The Fock space thus described gives the left action (or action onto the right) of the algebra $\mathcal{H}[a, b]$. The Fock space which provides the right action (or action onto the left) can be specified as the conjugation of the above, i.e. $\mathcal{F}^{*}[a, b]$.

It remains to specify the correlation functions for operators acting on the Fock spaces $\mathcal{F}[a, b]$ and $\mathcal{F}^{*}[a, b]$, or using more precise mathematical terminology, the pairing $\mathcal{F}[a, b] \otimes \mathcal{F}^{*}[a, b] \rightarrow C$. This is given by the following three steps. First, we fix the normalization for the vacuum vectors as follows,

$$
\left({ } _ { \alpha } \left\langle0\left|\otimes_{\beta}\langle 0|\right)\left(|0\rangle_{\alpha} \otimes|0\rangle_{\beta}\right)=1 .\right.\right.
$$

Next, for any two vectors

$$
\begin{aligned}
& \left\langle v_{f_{i}}\right|={ }_{\alpha}\langle 0| \otimes_{\beta}\langle 0| \int_{\epsilon}^{+\infty} d \lambda f_{i}(\lambda) X_{i}(\lambda), \\
& \left|v_{g_{j}}\right\rangle=\int_{-\infty}^{-\epsilon} d \mu g_{j}(\mu) X_{j}(\mu)|0\rangle_{\alpha} \otimes|0\rangle_{\beta}
\end{aligned}
$$


where $X_{i, j}(\lambda)$ are operators acting on the Fock spaces $\mathcal{F}[a, b]$ and $\mathcal{F}^{*}[a, b]$ satisfying

$$
\begin{aligned}
X_{i, j}(\lambda)|0\rangle_{\alpha} \otimes|0\rangle_{\beta} & =0=_{\alpha}\langle 0| \otimes_{\beta}\langle 0| X_{i, j}(-\lambda), \quad(\lambda>0) \\
{\left[X_{i}(\lambda), X_{j}(\mu)\right] } & =x_{i j}(\lambda) \delta(\lambda+\mu), \quad\left(x_{i j}(\lambda) \text { regular at } \lambda=0\right),
\end{aligned}
$$

with $f_{i}(\lambda)$ and $g_{j}(\lambda)$ both analytic in a small neighborhood of $\lambda=0$ except at $\lambda=0$ where they have simple poles, we define the inner product as follows:

$$
\left\langle v_{f_{i}} \mid v_{g_{j}}\right\rangle=\int_{C} \frac{d \lambda \ln (-\lambda)}{2 \pi i} f_{i}(\lambda) x_{i j}(\lambda) g_{j}(-\lambda)
$$

where $C$ is an integration contour which goes from infinity to zero above the positive real $\lambda$-axis, surrounding the origin counterclockwise, and going to infinity again below the positive real $\lambda$-axis. This particular kind of regularization has already been used in [7, 4]. Last, for 'multi-particle' states like $\left\langle v_{f_{i_{1}}, \ldots, f_{i_{k}}}\right|$ and $\left|v_{g_{j_{1}}, \ldots, g_{j_{k}}}\right\rangle$ we apply the Wick theorem.

Having provided all the necessary tools for defining the free boson representation, we now introduce the notation

$$
\begin{aligned}
\varphi(u) & =\int_{\lambda \neq 0} d \lambda e^{i \lambda u} a(\lambda), \\
\phi(u) & =\int_{\lambda \neq 0} d \lambda e^{i \lambda u} b(\lambda),
\end{aligned}
$$

where $\int_{\lambda \neq 0} d \lambda$ means the integration over the whole real $\lambda$ axis except the point $\lambda=0$, i.e.

$$
\int_{\lambda \neq 0} d \lambda=\lim _{\epsilon \rightarrow 0^{+}}\left(\int_{-\infty}^{-\epsilon} d \lambda+\int_{\epsilon}^{+\infty} d \lambda\right) .
$$

We then have

Proposition 3.1 The following expressions give a free boson realization of the algebra $\mathcal{A}_{\hbar, \eta}\left(\right.$ osp $\left.(2 \mid 2)^{(2)}\right)$ at $c=1$ :

$$
\begin{aligned}
E(u) & =e^{\gamma_{E}-\ln \eta}: \exp \left[\frac{i \pi}{2}\left(\frac{1}{p_{\alpha}} P_{\alpha}+\frac{1}{p_{\beta}} P_{\beta}\right)+\left(p_{\alpha} Q_{\alpha}+p_{\beta} Q_{\beta}\right)\right] \exp \hbar[\varphi(u)]:, \\
F(u) & =e^{\gamma_{E}-\ln \eta}: \exp \left[\frac{i \pi}{2}\left(\frac{1}{p_{\alpha}} P_{\alpha}-\frac{1}{p_{\beta}} P_{\beta}\right)+\left(p_{\alpha} Q_{\alpha}+p_{\beta} Q_{\beta}\right)\right] \exp \hbar[\phi(u)]:, \\
H^{ \pm}(u) & =: \exp \left[i \pi\left(\frac{1}{p_{\alpha}} P_{\alpha}\right)+2\left(p_{\alpha} Q_{\alpha}+p_{\beta} Q_{\beta}\right)\right] \exp \hbar[\varphi(u \pm i \hbar / 4)+\phi(u \mp i \hbar / 4)]:,
\end{aligned}
$$

where $p_{\alpha}, p_{\beta}$ are two arbitrary nonzero constants and $\gamma_{E}$ is the Euler constant $\gamma_{E}=0.57721566 \ldots$. 
The proof is by straightforward calculation using the Fock space conventions above. The following formulae play crucial roles:

$$
\begin{aligned}
\int_{C} \frac{d \lambda \ln (-\lambda)}{2 \pi i \lambda} \frac{e^{-x \lambda}}{1-e^{-\lambda / \eta}} & =\ln \Gamma(\eta x)+\left(\eta x-\frac{1}{2}\right)\left(\gamma_{E}-\ln \eta\right)-\frac{1}{2} \ln (2 \pi), \\
\Gamma\left(\frac{1}{2}-x\right) \Gamma\left(\frac{1}{2}+x\right) & =\frac{\pi}{\cos \pi x} .
\end{aligned}
$$

\subsection{Free boson representations of $U_{q}\left(\operatorname{osp}(2 \mid 2)^{(2)}\right)$}

In this subsection we carry out the same practice as in the last subsection without assuming the relation $1 / \eta^{\prime}=1 / \eta+\hbar$. As we shall see, this yields a representation of the algebra $U_{q}\left(\operatorname{osp}(2 \mid 2)^{(2)}\right)$ at $\gamma=q^{1 / 2}$. Below we give some of the details.

We introduce two Heisenberg algebras $\tilde{\mathcal{H}}_{\alpha}, \tilde{\mathcal{H}}_{\beta}$, not to be confused with those of the last subsection, defined respectively by

$$
\begin{aligned}
{[\alpha(\lambda), \alpha(\mu)] } & =A(\lambda) \delta(\lambda+\mu), \\
{[\beta(\lambda), \beta(\mu)] } & =B(\lambda) \delta(\lambda+\mu), \\
{[\alpha(\lambda), \beta(\mu)] } & =0, \\
(\lambda & \neq \mu)
\end{aligned}
$$

where $A(\lambda)$ and $B(\lambda)$ are given by

$$
\begin{aligned}
& A(\lambda)=\frac{\lambda}{4 \cosh \frac{\hbar \lambda}{2}+2}, \\
& B(\lambda)=-\frac{\lambda\left(\operatorname{csch}^{2} \frac{\lambda}{2 \eta} \sinh ^{2} \hbar \lambda+2 \cosh \hbar \lambda+1\right)}{4 \cosh \frac{\hbar \lambda}{2}+2} .
\end{aligned}
$$

The zero mode operators remain as in the last subsection:

$$
\begin{aligned}
& {\left[P_{\alpha}, Q_{\alpha}\right]=1,} \\
& {\left[P_{\beta}, Q_{\beta}\right]=1,} \\
& {\left[P_{\alpha}, Q_{\beta}\right]=\left[P_{\beta}, Q_{\alpha}\right]=0 .}
\end{aligned}
$$

Let

$$
\begin{aligned}
X_{a}(\lambda) & =\frac{1}{\lambda}\left(\operatorname{csch} \frac{\lambda}{2 \eta} \sinh \hbar \lambda+2 \cosh \frac{\hbar \lambda}{2}+1\right), \\
X_{b}(\lambda) & =\frac{1}{\lambda}\left(\operatorname{csch} \frac{\lambda}{2 \eta} \sinh \hbar \lambda-2 \cosh \frac{\hbar \lambda}{2}-1\right) \\
Y_{a}(\lambda) & =Y_{b}(\lambda)=\frac{1}{\lambda}
\end{aligned}
$$


and define

$$
\begin{aligned}
a(\lambda) & =X_{a}(\lambda) \alpha(\lambda)+Y_{a}(\lambda) \beta(\lambda) \\
b(\lambda) & =X_{b}(\lambda) \alpha(\lambda)+Y_{b}(\lambda) \beta(\lambda), \\
(\lambda & \neq \mu)
\end{aligned}
$$

so that the corresponding commutation relations read:

$$
\begin{aligned}
{[a(\lambda), a(\mu)] } & =-\frac{1}{\lambda}\left(1+\frac{\sinh \hbar \lambda}{\sinh \frac{\lambda}{2 \eta}}\right) \delta(\lambda+\mu), \\
{[b(\lambda), b(\mu)] } & =-\frac{1}{\lambda}\left(1-\frac{\sinh \hbar \lambda}{\sinh \frac{\lambda}{2 \eta}}\right) \delta(\lambda+\mu), \\
{[a(\lambda), b(\mu)] } & =[b(\lambda), a(\mu)]=\frac{2}{\lambda} \cosh \frac{\hbar \lambda}{2} \delta(\lambda+\mu), \\
(\lambda & \neq \mu) .
\end{aligned}
$$

We introduce the free bosonic fields $\varphi(u)$ and $\phi(u)$ as in (19) but using the Heisenberg algebras described in this subsection.

Proposition 3.2 The following expressions give a free boson realization of the algebra $U_{q}\left(\operatorname{osp}(2 \mid 2)^{(2)}\right)$ currents (8-14) at $\gamma=q^{1 / 2}$ :

$$
\begin{aligned}
X^{+}(z) & =e^{\gamma_{E}-\ln \eta} \frac{1}{\sqrt{2} z}: \exp \left[\frac{i \pi}{2}\left(\frac{1}{p_{\alpha}} P_{\alpha}+\frac{1}{p_{\beta}} P_{\beta}\right)+\left(p_{\alpha} Q_{\alpha}+p_{\beta} Q_{\beta}\right)\right] \\
& \times \exp \left[\varphi\left(\frac{\ln z}{2 \pi \eta}\right)\right]:, \\
X^{-}(z) & =e^{\gamma_{E}-\ln \eta} \frac{1}{\sqrt{2} z}: \exp \left[\frac{i \pi}{2}\left(\frac{1}{p_{\alpha}} P_{\alpha}-\frac{1}{p_{\beta}} P_{\beta}\right)+\left(p_{\alpha} Q_{\alpha}+p_{\beta} Q_{\beta}\right)\right] \\
& \times \exp \left[\phi\left(\frac{\ln z}{2 \pi \eta}\right)\right]:, \\
\psi^{ \pm}(z) & =\frac{(2 \pi)^{2} i \eta\left(q-q^{-1}\right)}{\ln q}: \exp \left[i \pi\left(\frac{1}{p_{\alpha}} P_{\alpha}\right)+2\left(p_{\alpha} Q_{\alpha}+p_{\beta} Q_{\beta}\right)\right] \\
& \times \exp \left[\varphi\left(\frac{\ln z q^{ \pm 1 / 4}}{2 \pi \eta}\right)+\phi\left(\frac{\ln z q^{\mp 1 / 4}}{2 \pi \eta}\right)\right]:,
\end{aligned}
$$

where $p_{\alpha}, p_{\beta}$ are two arbitrary nonzero constants, $\gamma_{E}$ is the Euler constant, and $q$ and $z$ are related to the parameters $\eta$ and $\hbar$ via $q=e^{2 \pi i \eta \hbar}$ and $z=e^{2 \pi \eta u}$.

Remark 3.3 We can set the parameter $\eta$ in the above free boson representation to any fixed value without changing the representation itself. In this sense, the parameter $\eta$ can be thought of as redundant. Indeed, the algebra $U_{q}\left(\operatorname{osp}(2 \mid 2)^{(2)}\right)$ contains only one deformation parameter, and its representation must necessarily contain no extra parameters. 
Remark 3.4 According to the Footnote 1, the value of $\gamma=q^{1 / 2}$ corresponds to $c=1 / 2$ in [B] . Thus the free boson representation given in Proposition 3.9 is somehow not the most interesting one - remember that for usual affine Lie (super)algebras, only representations at integer values of c have received attention, because only these representations are known to be unitary.

Remark 3.5 In [8], a free boson representation of the $U_{q}\left(\operatorname{osp}(2 \mid 2)^{(2)}\right)$ currents (8-14) at $\gamma=q$ (i.e. $c=1$ ) was given. However, that representation does not have a well-defined limit as $q \rightarrow 1$ and hence the authors of that paper called their representation a 'nonclassical' one. One can verify that our representation does have a well-defined limit at this value of deformation parameter.

Although the representation of $U_{q}\left(\operatorname{osp}(2 \mid 2)^{(2)}\right)$ at $\gamma=q^{1 / 2}$ is not of the most interesting class, we could, however, use the same method to construct 'interesting' representations. Now, instead of (20-22), we introduce the following set of bosonic algebras:

$$
\begin{aligned}
{\left[a_{n}, a_{m}\right] } & =-\frac{1}{n}\left(1+(-q)^{-n}\right) \delta_{n+m, 0}, \\
{\left[b_{n}, b_{m}\right] } & =-\frac{1}{n}\left(1-(-q)^{-n}\right) \delta_{n+m, 0}, \\
{\left[a_{n}, b_{m}\right] } & =\left[b_{n}, a_{m}\right]=\frac{1}{n}\left(q^{n}+q^{-n}\right) \delta_{n+m, 0},
\end{aligned}
$$

together with the zero mode operators:

$$
\begin{aligned}
{\left[P_{a}, Q_{a}\right] } & =1, \\
{\left[P_{b}, Q_{b}\right] } & =1, \\
{\left[P_{a}, Q_{b}\right] } & =\left[P_{b}, Q_{a}\right]=0 .
\end{aligned}
$$

These bosonic commutation relations can also be realized in the tensor product of Fock spaces of two commuting sets of Heisenberg algebras, though we omit this here.

Defining

$$
\begin{aligned}
\varphi(z) & =\sum_{n \neq 0} a_{n} z^{-n}+P_{a} \ln z+2 Q_{a}+2 Q_{b}, \\
\phi(z) & =\sum_{n \neq 0} b_{n} z^{-n}-\left(P_{a}+P_{b}\right)(\ln z+i \pi / 2)-2\left(Q_{a}-Q_{b}\right),
\end{aligned}
$$

we can easily prove

Proposition 3.6 The following bosonic expressions give a free boson representation of $U_{q}\left(\operatorname{osp}(2 \mid 2)^{(2)}\right)$ currents at $\gamma=q$ :

$$
\begin{aligned}
E(z) & =: \exp \varphi(z): \\
F(z) & =: \exp \phi(z): \\
H^{ \pm}(z) & =: E\left(z q^{ \pm 1 / 2}\right) F\left(z q^{\mp 1 / 2}\right): .
\end{aligned}
$$

Again, this free boson representation is well defined as $q \rightarrow 1$. 


\section{Concluding Remarks}

The result in this article provides an example of a two-parameter deformed quantum current algebra with the structure of an infinite Hopf family of superalgebras and associated with a nonsimply-laced and twisted root system. To the authors' knowledge, this is the first example of this kind and hence a useful hint at the final classification of all such algebras.

As mentioned in the introduction, algebras with the structure of an infinite Hopf family of (super)algebras have so far been studied only in current realizations, and this limitation has made understanding the relationship between these algebras and the quasi-Hopf algebras a difficult task. Meanwhile, in most physical applications, quantum-deformed algebras are best formulated in terms of the Yang-Baxter relations (the celebrated ' $R L L$ ' relations). It is expected that if such a realization for the algebras of the present kind can be achieved, then many of the unsolved problems mentioned above could be treated more easily. The $R L L$ realization of the two-parameter deformed algebras with the structure of infinite Hopf families of algebras is currently under investigation.

Acknowledgement: L. Zhao would like to thank the Department of Mathematics, University of York for hospitality. Discussions with Xiang-Mao Ding and financial support from the Royal Society of London, the UK PPARC and the National Natural Science Foundation of China are also warmly acknowledged.

\section{References}

[1] Drinfeld, V.G., Quasi-Hopf algebras, Leningrad Math. J. 1 (1990)1419.

[2] Feigin B., Frenkel E., Quantum $W$-alebras and Elliptic algebras, q-alg/9508009, Commun. Math. Phys. 178 (1996) 653.

[3] Hou, B.-Y., Yang, W.-L., Dynamically twisted algebra $A_{q, p, \hat{\pi}}\left(\widehat{g l}_{2}\right)$ as current algebra generalizing screening currents of $q$-deformed Virasoro algebra, Preprint q-alg/9709024.

[4] Hou, B.-Y., Zhao, L., Ding, X.-M., The algebra $\mathcal{A}_{\hbar, \eta}(\hat{g})$ and infinite Hopf family of algebras, Preprint q-alg/9703046, J. Geom. Phys. 27 (1998) 249.

[5] Hou, B.-Y., Zhao, L., Ding, X.-M., Infinite Hopf family of elliptic algebras and bosonization, J. Phys. A: Math. Gen. 32 (1999) 1951.

[6] Jimbo, M., Konno, H., Odake, S., Shiraishi, J., Quasi-Hopf twistors for elliptic quantum groups, q-alg/9712029.

[7] Khoroshkin, S., Lebedev, D., Pakuliak, S., Elliptic algebra $A_{p, q}\left(\widehat{s l}_{2}\right)$ in the scaling limit, Preprint q-alg/9702002. 
[8] Yang, W.L. and Zhang, Y.Z., Drinfeld Basis And a Nonclassical Free Boson Representation of Twisted Quantum Affine Superalgebra $U_{q}\left[\operatorname{sos}(2 \mid 2)^{(2)}\right]$, preprint nath.QA/9904017.

[9] Zhao, L. and Ding, X.M., On two-parameter deformations of $\operatorname{osp}(1 \mid 2)^{(1)}$, Lett. Math. Phys. 51 , No.3 (2000) 